\title{
EXTRAMEDULLARY PLASMACYTOMA OF NOSE AND PARANASAL SINUSES, TWO CASE REPORTS
}

Souvagini Acharya, Manas Ranjan Satpathy

1. Assistant Professor. Department of ENT, VSS Medical College, Burla, Odisha.

2. Assistant Surgeon. VSS Medical College, Burla, Odisha.

\section{CORRESPONDING AUTHOR:}

Dr. Souvagini Acharya

Assistant Professor, Department Of ENT, V.S.S. Medical College, Burla, E-mail: drs.acharya@yahoo.co.in Ph: 00919437222414.

ABSTRACT: Extramedullary plasmacytoma is a rare malignant plasma cell tumor. We report two extremely rare cases of extramedullary plasmacytoma, one of the maxillary sinus and the other of the nasal cavity. A $57 \mathrm{yr}$ old man presented with swelling of right maxilla and hard palate. CT scan revealed right maxillary mass with inferior and lateral extension. Another $63 \mathrm{yr}$ old woman presented with mass in left nostril with a huge swelling to the left of nose. Histopathology examination of both the masses revealed abundant plasma cells and diagnosed as extramedullary plasmacytoma. Both patients were planned for radiotherapy.

INTRODUCTION: Extramedullary plasmacytoma is a localized collection of monoclonal plasma cells in an extra skeletal site. Extramedullary plasmacytoma (EMP) represents approximately $3 \%$ of all plasma cell neoplasms and $<1 \%$ of all head and neck tumors (1). The tumours arise predominantly in the sub mucosal tissues of the nasal cavity, paranasal sinus, nasopharynx, oropharynx and larynx. The nasal cavity and nasal septum are probably the most common locations.(2) The incidence in males is three times that of females. The most affected age group is 50-70 years. It is usually solitary or localized, but $10 \%$ of the patient have multiple site involvement.(3) We present two cases of extramedullary plasmacytoma. One of the right maxillary sinus and the other of the left nasal cavity.

\section{CASE REPORT:}

CASE 1: A $57 \mathrm{yr}$ old male farmer presented to ENT OPD of VSS medical college Burla with swelling of right side of face for six months, nasal blockage for four months, difficulty in chewing food on right side for three months and loosening and falling of right upper teeth. He also had dull pain over the right side cheek. He also experienced a loss of appetite and loss of weight in the past two months.

Physical examination revealed a diffuse swelling of the right cheek with induration of the overlying skin. It extended superiorly up to the lower eyelid and laterally causing a bulge in the parotid region. On anterior rhinoscopy, there was no obvious mass in the right nostril, but the lateral wall was pushed medially. On examination of the oral cavity, there was gross swelling of the upper alveolus with disarray of teeth arrangement. There was also breech of mucosa over it exposing the tumor mass at some places. 
CT scan revealed a $7 \mathrm{~cm} \times 7 \mathrm{~cm} \times 8 \mathrm{~cm}$ mass occupying the right maxillary sinus. There was bony erosion inferiorly into the oral cavity and laterally into cheek. An incisional biopsy was taken from the tumour mass intraorally through the exposed area in the upper alveolus. The histopathological examination revealed the presence of abundant plasma cells with kappa light chain restriction. Bone marrow examination was normal. The clinical features and histopathological findings were consistent with extramedullary plasmacytoma of maxillary sinus. A systemic work up including blood profile, renal and liver function tests, serum and urinary protein electrophoresis, serum immunoglobulin level, skeletal survey was done to exclude a systemic disease like multiple myeloma. The patient was planned for radiotherapy.

CASE 2: A 63 yr old housewife complained of a slowly growing mass in the left nostril for eight months and swelling over left side of nose for four months. She has also been experiencing recurrent scanty blood stained nasal discharge from left nostril over last three months.

Examination revealed a $6 \mathrm{~cm} \times 6 \mathrm{~cm}$ swelling on the left side of nose. It extended laterally to almost block the vision of left eye and superiorly touched the upper eyelashes. On anterior rhinoscopy, a red, firm, smooth surfaced mass was seen filling the left nostril. Punch biopsy was taken from the left nostril mass. Histopathology again showed large population of plasma cells. Bone marrow examination was normal. Blood profile, renal and liver function tests, serum and urinary protein electrophoresis, serum immunoglobulin and skeletal survey was within normal limit.So it was consistent with extramedullary plasmacytoma of nasal cavity. She was also planned for radiotherapy.

DISCUSSION: Extramedullary plasmacytoma arising from nose and paranasal sinuses are uncommon and are easily confused with squamous cell carcinoma which is more commonly encountered. Therefore our initial provisional diagnosis was squamous cell carcinoma in both the cases. However, histopathology revealed extramedullary plasmacytoma, a relatively rare entity.

Extramedullary plasmacytoma is a localized collection of monoclonal plasma cells in an extraskeletal site. It is more common in males with a median age of 50-70 yrs. Approximately $80 \%$ are localized in the submucosa of the upper respiratory tract including nasal cavity, septum, nasopharynx and maxillary sinus.(4) Other sites in the head and neck that have been affected include the thyroid gland, soft tissues of the anterior cervical region, parotid gland, tonsil, oropharynx, larynx, orbit, choroid and eyelid. Korolkowa et al reported that $40 \%$ occur in the nasal cavity and paranasal sinuses, $20 \%$ in the nasopharynx, and $18 \%$ in the oropharynx. Approximately $10 \%$ of extramedullary plasmacytomas have multiple sites of involvement.(5)

The clinical presentations of extramedullary plasmacytoma and carcinoma of maxillary sinus are almost similar. The diagnosis of plasmacytoma is based on and confirmed with histology and immunohistochemistry. There is dense, homogeneous infiltrate of plasma cells. Amyloid deposition may be seen in 15\%-38\% of extramedullary plasmacytoma.(6) The commonest immunoglobulin expressed by the tumour cells is IgG with kappa chain restriction. Plasmacytoma can be graded low (grade 1), intermediate (grade 2) and high grades (grade 3), based on the cellular atypia. Based on the serum, urine electrophoresis, bone scan, bone marrow examination and radiological assessment, extramedullary plasmacytoma can be staged according to the spread of the disease. Stage I is disease confined to one site. Stage II includes tumours with local extension of lymph node involvement. Stage III has metastatic spread. 
Localised extramedullary plasmacytoma is highly radiosensitive. According to the guidelines published in British Journal of Haematology, the recommended primary treatment for localised extramedullary plasmacytoma is radical radiotherapy. For generalised extramedullary plasmacytoma, chemotherapy is advisable.(7).

For extramedullary plasmacytoma localized to head and neck not extending to floor of anterior and middle cranial fossa and orbit radiotherapy is the treatment of choice while surgery is limited to biopsy and excision of residual mass(8). A follow-up examination with CT is suggested to determine recurrence and to rule out simultaneous lesions in different sites of the head and neck, which are known to occur in 10 to $20 \%$ of patients.

We treated both the patients with radical radiotherapy and both of them are doing well till now, three years after completion of radiotherapy.

\section{REFERENCES}

1. Miller FR, Lavertu P, Wanamaker JR, et al. Plasmacytomas of the head and neck. Otolaryngol Head Neck Surg 1998;119:614-8.

2. Paris J, Dessi P, Moulin G, et al. [Extramedullary plasmacytoma of the nasal cavity: A case report]. Rev Laryngol Otol Rhinol (Bord) 1999;120:343-5.

3. Gonzalez Aguilaro O, Dragosky M, Pardo HA, et al. [Single extramedullary plasmacytoma of the nose]. Acta Otorrinolaringol Esp 2000;51:348-52.

4. Courtmans I, Pigeolet Y, Hedayat AH, Vilain J. Upper airways locations of plasmacytoma. Acta Otorhinolaryngol Belg 2000;54:487-90.

5. Windfuhr JP, Ott G. Extramedullary plasmacytoma manifesting as a palpable mass in the nasal cavity. Ear Nose Throat J 2002; 81:110-4.

6. Korolkowa 0, Osuch-Wójcikiewicz E, Depta a A, Suleiman W. [Extramedullary plasmacytoma of the head and neck]. Otolaryngol Pol 2004; 58:1009-12. Polish.

7. Soutar R, Lucraft H, Jackson G, et al. Guidelines on the diagnosis and management of solitary plasmacytoma of bone and solitary extramedullary Splasmacytoma. $\mathrm{Br} \mathrm{J}$ Haematol 2004; 124:717-26.

8. Soo G, Chan A,Lam D, Abdullah V,Van Haselt CA.Extramedulary nasal plasmacytoma an unusual clinical entity.ENT journal 1996;75:171-3..

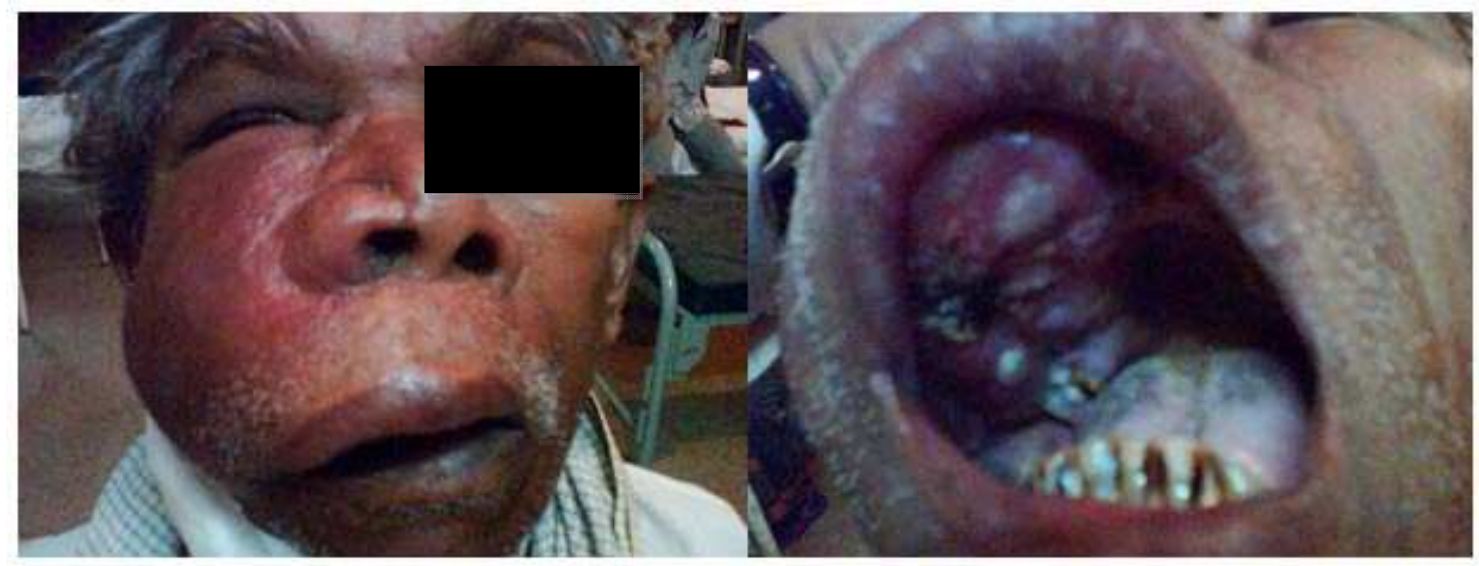

CASE 1: View from front and intraoral view showing palate-alveolar extent of the tumor, from where the biopsy was taken. 


\section{CASE REPORT}

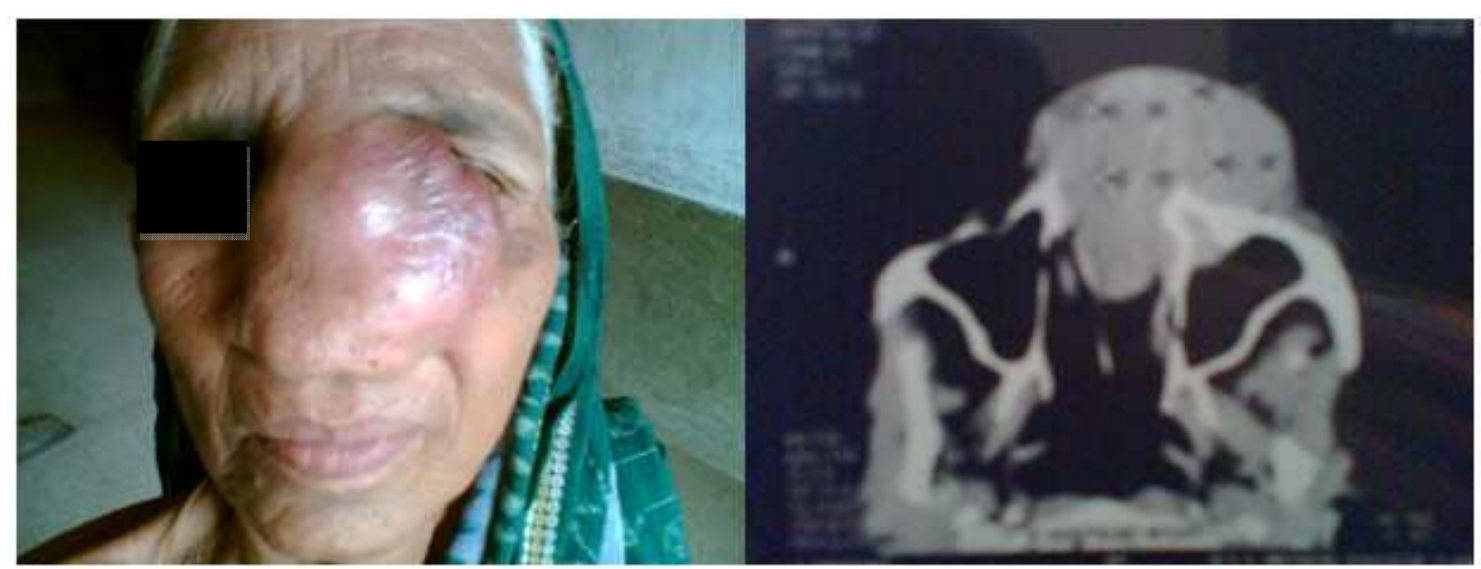

CASE 2: View from front and her CT scan showing tumour involving nasal cavity and external soft tissue extension. Maxillary sinus is not involved.

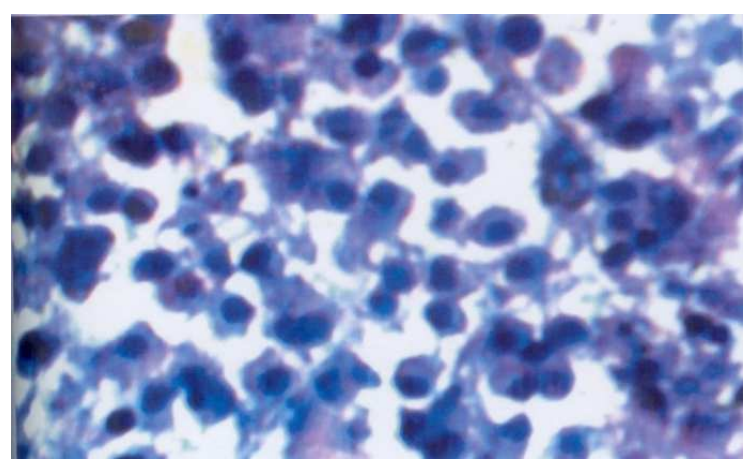

CASE 2: Histopathology of the punch biopsy showing plasma cells with varying degrees of maturation. 